\title{
MOVILIDAD ESPACIAL DE LA POBLACIÓN Y CAMBIOS RECIENTES DEL MODELO DE DENSIDAD EN CIUDADES DEL SISTEMA URBANO CHILENO ${ }^{1}$
}

\author{
Severino Escolano Utrilla \\ Departamento de Geografía y Ordenación del Territorio \\ Universidad de Zaragoza (España) \\ severino@unizar.es \\ Jorge Ortiz Véliz \\ Departamento de Geografía \\ Universidad de Chile (Chile) \\ jortiz@uchile.es
}

\begin{abstract}
This paper deals with some changes that recently have taken place in the density population model of some cities belonging to the Chilean urban system. The main features of the new model, compare with the previous compact city one, are: fragmentation in several parts, reorganization around many spatial modes and density average decrease. One consequence of the population redistribution in the urban space is the increase on the distance and time average between the residence places and the urban center or the functional areas.

The immediate causes of the population distribution model restructuring are found in the fragmentation of the urban builts space and in the successive intraurban migration waves, which predominant direction is from the center to the increasingly far away peripheries. We also state that some changes on the population density model are common to many cities and, therefore, independent to its size.
\end{abstract}

Keywords: Density of urban population, urban fragmentation, urban migrations.

Resumen: Este artículo trata sobre algunos cambios recientes habidos en el modelo de densidades de población de algunas ciudades del sistema urbano chi-

\footnotetext{
1 Proyecto FONDECYT n. ${ }^{\circ} 7040151$; proyecto FONDECYT n. ${ }^{\circ} 1040707$

Recibido: 1-9-2005. Aceptado: 30-5-2006.
} 
leno. Los rasgos fundamentales del nuevo modelo, en relación con el de la ciudad compacta previa, son: disgregación en múltiples fragmentos, reorganización en torno a múltiples modas espaciales y descenso de la densidad media. Una consecuencia de la redistribución de la población en el espacio urbano es el aumento de los recorridos medios entre el lugar de residencia y el centro urbano o los centros funcionales.

Las causas inmediatas de la reestructuración del modelo de distribución de población se encuentran en la fragmentación del espacio urbano construido y en las sucesivas oleadas de las migraciones intraurbanas, cuya dirección predominante es desde el centro hacia las periferias cada vez más alejadas. También mantenemos que algunos cambios del modelo de densidades de población son comunes a muchas ciudades y, por tanto, independientes del tamaño de la ciudad.

Palabras clave: Densidad de población urbana, fragmentación urbana, migraciones intraurbanas.

\section{Introducción: el insostenible e incontenible ímpetu del crecimiento urbano}

Tal vez el cambio sea el rasgo más persistente de la evolución urbana; sin embargo, es posible identificar períodos de mayor estabilidad frente a otros caracterizados por intensas modificaciones, como sucede desde hace treinta años. En efecto, durante este tiempo, la actuación convergente en el espacio urbano de múltiples procesos de naturaleza diversa, ha producido transformaciones de magnitud que han alterado la extensión, las estructuras y el paisaje de las ciudades. Los avatares del devenir urbano reciente se relacionan con otros cambios de envergadura que han sacudido los cimientos de la organización social, la cultura, los valores, la tecnología...

La vitalidad tan extraordinaria mostrada por el fenómeno urbano desde mediados del siglo XX en América del Sur, ha colocado a la región entre las más urbanizadas del mundo. En 2003 la población urbana de Sudamérica alcanzó el 81,1\% de la población total (más de 294 millones de personas), tasa que duplica casi la correspondiente a 1950 (43,7\%) (UN, WUP. The 2003 Review). Una nota distintiva de la urbanización sudamericana se encuentra en el gran peso demográfico $y$, por supuesto, económico, político y funcional, de las grandes urbes metropolitanas (mayores de 5 millones de habitantes) que concentran cerca del $21 \%$ de la población total (UN, WUP., op. cit.).

El espacio urbano se ha convertido en un gigantesco tablero en que despliegan sus estrategias numerosos agentes, individuos y corporaciones, que actúan a micro- 
escala o a escala mundial. Es notorio que los efectos asociados a los procesos de reestructuración no se manifiestan de manera similar en todas las ciudades del subcontinente, en las de un país, ni siquiera en núcleos urbanos vecinos. Más bien al contrario, su desarrollo y formas presentan disparidades y contradicciones producidas por la compleja combinatoria de factores locales y supralocales. No obstante, también se constatan características comunes, especialmente entre las grandes ciudades, patentes en los modos dominantes que adopta el crecimiento urbano. Entre otras, destacan: la incorporación selectiva y fragmentada de grandes porciones periféricas al espacio urbano consolidado; la carencia de servicios y equipamientos y el desigual acceso a los mismos entre clases sociales y áreas de las ciudades; la fragmentación física, funcional y social; la baja accesibilidad de extensas zonas urbanas; la aparición de enormes centros funcionales en la periferia de las ciudades; el crecimiento de la dispersión urbana; la insuficiencia generalizada de recursos públicos. (Arraigada y Rodríguez, 2003; Balbo, Jordán y Simioni, 2003; Kaztman, 2003; Arraigada, 2000; Clichevsky, 2000; de Mattos, 2002; Prevot, 1999).

En conjunto, el desarrollo del conglomerado de fuerzas que actúan sobre el espacio urbano ha dado lugar a ciudades más complejas, pero más ineficientes, cuyo funcionamiento ocasiona graves daños ambientales, deteriora partes del espacio construido y fomenta y expande las desigualdades socioterritoriales, mediante mecanismos de exclusión social y reproducción de la pobreza.

En consecuencia, es evidente que la mejora del bienestar de la población y de la capacidad y eficiencia productivas, la conservación eficaz del medio ambiente y del patrimonio, así como la construcción de una sociedad más equitativa, pasan por hacer de las ciudades espacios más adecuados para la vida y convivencia urbanas.

Para alcanzar esta meta es necesario perfeccionar el conocimiento científico de los procesos que guían los cambios. Ello requiere datos más abundantes, exactos, precisos y más apropiados, así como renovados métodos y herramientas de análisis. Pero sobre todo, se precisan proyectos, políticas urbanas y recursos encaminados a construir ciudades más sostenibles, desde el punto de vista social, económico y ambiental.

\section{Objetivos e hipótesis. La dispersión de la población es connatural a la mercantilización de la ciudad}

El estudio de cualquier componente de la ciudad o de una parte de la misma, y no digamos de una ciudad completa, reviste dificultades de variado tipo, debidas a la compleja y densa red de relaciones mutuas que atan a la ciudad con otras dimensiones socioeconómicas. De aquí que, en cualquier investigación, sea tarea previa obli- 
gada delimitar el ámbito de validez de las conclusiones mediante los oportunos recortes temáticos, espaciales y temporales, y la definición de las unidades de análisis apropiadas.

Este trabajo parte del reconocimiento de que los nuevos modos del crecimiento urbano generan, necesariamente, movimientos espaciales intraurbanos de la población cuyo vector resultante toma la dirección centro-periferia. Estos desplazamientos superan en volumen, en las grandes ciudades, a los tradicionales campo-ciudad; su origen, destino y magnitud obedecen, entre otras razones, a la creación de fragmentos urbanos separados, a veces, del tejido urbano preexistente por una distancia considerable; a los mecanismos de revalorización del suelo urbano; a las tremendas desigualdades en la distribución de la renta; y a la promoción y difusión de modelos urbanos foráneos (casa aislada con jardin en condominio cerrado) que consumen mucho suelo y usan de modo ineficiente las infraestructuras.

La relocalización de la población a través de las migraciones intraurbanas es la causa principal de los cambios en la estructura del modelo de distribución de la densidad de población, que pasa desde el tradicional, propio de ciudades compactas (gradiente decreciente desde el centro hacia la periferia) a otro fragmentado, cuyos valores más elevados se disponen en sectores discontinuos en torno al centro.

Un corolario del incremento de la dispersión de la población es el aumento de la distancia promedio agregada que los compradores y usuarios han de recorrer para adquirir bienes y servicios propios del centro de la ciudad. En consecuencia, la ciudad, considerada como un sistema funcional, pierde eficacia, pues se acrecienta el consumo de energía per capita, la presión sobre las infraestructuras y medios de transporte, se alarga la duración de los viajes, se multiplican los atascos, se agrava la contaminación atmosférica...

Por otra parte, aunque no es objetivo de este artículo, las migraciones intraurbanas constituyen la vía preferente a través de la cual se acentúa la segregación debido, por un lado, al modo de ocupación del suelo en grandes lotes homogéneos, y por otro, a la selección demográfica y socioeconómica de los migrantes en función de los lugares de origen y destino.

Aquí se analizan los patrones espaciales de la movilidad intraurbana entre 1997 y 2002, a partir los últimos datos censales publicados, y se construye y caracteriza el modelo de densidad de población. El estudio comprende el espacio metropolitano del Gran Santiago, donde se han investigado y verificado los procesos descritos, y dos ciudades medias con base económica diferente: La Serena, en la IV Región de Coquimbo, y Puerto Montt, capital de X Región de Los Lagos. La hipótesis implícita en esta muestra acepta que en las ciudades medias se reproducen, en general, procesos idénticos a los que operan en las grandes ciudades. No obstante, las diferencias de tamaño entre aquéllas y éstas hacen que la escala de los cambios y sus moda- 
lidades difieran en magnitud, formas y a veces en naturaleza. El propósito no es tanto medir las semejanzas y diferencias entre las tres ciudades como explorar el alcance y efectos de los cambios en el modelo de densidad.

\section{Métodos y datos}

La vía metodológica seguida en este trabajo es esencialmente exploratoria. Por un lado, la relación entre los patrones espaciales de las migraciones intraurbanas y el modelo de distribución de densidades se establece de forma directa mediante mapas. Por otro, se elaboran, describen y comparan, hasta donde es posible, la estructura de las densidades mediante procedimientos analíticos y gráficos.

El análisis de los flujos migratorios intraurbanos está limitado por las unidades espaciales de agregación de los datos. La información censal, obtenida a través del sistema REDATAM, sólo permite conocer la comuna de procedencia de los habitantes de cualquier delimitación estadístico-administrativa. Por esta razón, en las ciudades contenidas en una única comuna, como Puerto Montt y parcialmente La Serena, no es posible dar cuenta de la dinámica migratoria intraurbana, a diferencia de la capital nacional, ciudad conformada por 34 comunas urbanas.

La dispersión y fragmentación urbana se evalúan a través del modelo de densidades, de la medida de la compacidad del espacio urbano construido y de la distancia promedio agregada desde la residencia de la población hasta el centro de la ciudad.

La densidad se ha calculado por un procedimiento de filtrado espacial (kernel), cuyas especificaciones se detallarán más adelante. El modelo y su representación cartográfica son especialmente apropiados para generalizar datos como los de población, cuyas unidades de medida naturales son individuos discretos (personas) (Thurstain-Goodwin, 2003). La densidad obtenida por este método obvia algunas limitaciones inherentes al concepto habitual de densidad (ratio formado a partir de unidades administrativas arbitrarias), y otorga cierta continuidad espacial a este cociente, más acorde con las prácticas de uso del espacio urbano (Escolano, 2002).

La forma del espacio construido y su eficiencia se miden a través del índice rbo de Bertaud y Malpezzi (1999), y de la distancia media de la población al centro urbano. El primero relaciona la distancia promedio desde la residencia de la población a la localización del centro urbano (o de cualquier centro de comercio y servicios) con la misma magnitud de una figura ideal, un cilindro cuya altura es la densidad media y la superficie de la base es igual a la superficie del área de estudio. Su ecuación es: 


$$
\rho=\sum \mathrm{d}_{\mathrm{i}} \mathrm{p}_{\mathrm{i}} / \mathrm{PC}
$$

$\mathrm{d}_{\mathrm{i}}$ : distancia desde el centro funcional urbano (CBD) y la residencia de la población residente en $\mathrm{i}$.

$\mathrm{p}_{\mathrm{i}}$ : población del lugar i (portal, centroide de una manzana o de un sector urbano).

C: promedio de distancia de la población al centro de la base de un cilindro de superficie igual a la de la zona de estudio, y cuya densidad (altura del cilindro) es constante. Este valor, obtenido por cálculo integral, es aproximadamente $2 / 3$ del radio del círculo.

P: población total.

Los datos demográficos manejados proceden del censo de población y viviendas de 2002 (REDATAM). La delimitación espacial de las unidades administrativas es la establecida en la cartografía del Instituto Geográfico Nacional (INE), y los perímetros de las ciudades se han obtenido de fotogramas aéreos y de trabajos de campo.

Algunos resultados son orientativos más que significativos, pues dependen de las unidades empleadas y de la precisión de las medidas; por ejemplo, el índice rho está influido por el valor de la superficie de la ciudad, que no es fácil de determinar cuando, como es el caso, sus contornos son difusos.

La tecnología de los sistemas de información geográfica (SIG) ha sido utilizada con preferencia, tanto para integrar los datos y realizar cálculos analíticos como para elaborar las representaciones gráficas. Los mapas temáticos y los gráficos son documentos básicos en los que se fundamentan algunas interpretaciones contenidas en este trabajo.

Tabla 1. Dimensiones de investigación, variables y fuentes.

\begin{tabular}{|c|c|c|c|}
\hline $\begin{array}{l}\text { Dimensiones } \\
\text { de la investigación }\end{array}$ & $\begin{array}{l}\text { Variables } \\
\text { Indicadores }\end{array}$ & $\begin{array}{l}\text { Unidades } \\
\text { espaciales }\end{array}$ & $\begin{array}{l}\text { Fuentes } \\
\text { Observaciones }\end{array}$ \\
\hline $\begin{array}{l}\text { Patrones espaciales de } \\
\text { las migraciones } \\
\text { intraurbanas (1997- } \\
2002)\end{array}$ & $\begin{array}{l}\text {-Comuna de } \\
\text { residencia en } 1997\end{array}$ & $\begin{array}{l}\text {-Desde sector (la menor, } \\
\text { por debajo de la man- } \\
\text { zana urbana) hasta el } \\
\text { país completo }\end{array}$ & $\begin{array}{l}\text { Censos de población } \\
\text { y vivienda de } 2002, \\
1992 \text { y } 1982\end{array}$ \\
\hline $\begin{array}{l}\text { Intensidad y forma de } \\
\text { la localización } \\
\text { residencial de la } \\
\text { población en } 2002\end{array}$ & \begin{tabular}{|l}
-Residencia de la \\
población \\
-Distancia desde la \\
residencia al cen- \\
tro urbano \\
-Superficie urbana \\
-Densidad \\
-Rbo \\
-Distancia promedio
\end{tabular} & $\begin{array}{l}\text {-Centroides: } \\
\text { de manzanas (Puerto } \\
\text { Montt), de agrupacio- } \\
\text { nes de manzanas (La } \\
\text { Serena) y de distrito } \\
\text { (Santiago) } \\
\text {-Centro urbano: plaza } \\
\text { de Armas de cada ciu- } \\
\text { dad }\end{array}$ & $\begin{array}{l}\text {-Censos de población } \\
\text { y vivienda } \\
\text {-Cartografía base del } \\
\text { INE y del Ayto. de } \\
\text { Puerto Montt } \\
\text {-Imágenes aéreas del } \\
\text { sistema nacional de } \\
\text { información ambien- } \\
\text { tal (SINIA) }\end{array}$ \\
\hline
\end{tabular}




\section{Resultados: similares procesos de reestructuración, diferentes fases e intensidad}

Un recorrido, incluso apresurado, por las ciudades objeto de estudio evidencia que su crecimiento se ha producido por extensión del espacio construido más que por densificación del espacio preexistente. El examen posterior de los datos de densidad y el cotejo de planos y fotogramas aéreos corroboran esta primera impresión. No obstante, esta generalización debe de ser matizada, pues la renovación de los centros históricos y sus aledaños ha introducido cambios en los usos del suelo acompañados del incremento del volumen edificado: algunas casas tradicionales de una o dos plantas han sido sustituidas por edificios de cuatro o cinco plantas y en Santiago también por edificaciones de treinta pisos.

Otro aspecto fundamental asociado a los nuevos modos de crecimiento urbano, accesible también por simple observación, se encuentra en la discontinuidad espacial de los nuevos fragmentos periféricos. La tipología construida predominante consiste en casas unifamiliares, aunque en algunas ciudades como Puerto Montt, La Serena e incluso Santiago es tipo de edificación tradicional. Quizás, el cambio más importante sea el modo extensivo de ocupar el espacio, con desarrollos residenciales aislados (frecuentemente en condominios cerrados) que dejan intersticios monofuncionales de usos mixto (rural, actividades industriales, de comercio o servicios) o simplemente, permanecen vacios a la espera de su revalorización.

Esta forma de ocupación no sólo es más ineficiente, sino que además modifica el significado y función de ciertos elementos urbanos y la estructura de partes del tejido urbano; por ejemplo, el concepto de calle tal como se vive y materializa en el centro de la ciudad y de los barrios, se disuelve hasta devenir en mera vía de acceso.

Los cambios morfológicos señalados son un trasunto de los procesos esbozados, al tiempo que orientan otras modificaciones menos visibles en la distribución espacial de la población, pero igualmente decisivas en todos los órdenes de la vida urbana.

\subsection{Crecimiento demográfico, movilidad espacial de la población y extensión del espacio construido}

El crecimiento y la fragmentación del tejido urbano se deben a la intervención conjunta de un complejo de fuerzas que interactúan siguiendo una secuencia temporal y espacial determinada. Una parte de la expansión de la ciudad se justifica por el crecimiento demográfico y por la mejora necesaria del tamaño y de la calidad de las viviendas, pero otra, tal vez desmesurada, así como la localización periférica y ais- 
lada, responden a principios de maximización de plusvalías mediante creación de suelo urbano. Es obvio que el proceso, además de movilizar ingentes medios de promoción, impulsa el cambio de residencia desde el centro a los alrededores de la ciudad.

Los cambios estructurales recientes de las ciudades van asociados al aumento espectacular de la población urbana y a su pujanza económica y funcional. Los datos de las tablas 2 y 3 muestran que el crecimiento demográfico de las ciudades estudiadas ha sido más elevado que el del promedio del país, al menos desde la década de los 30 del pasado siglo (y también lo ha sido el de las capitales regionales). Hasta los 80, las tasas de crecimiento del Gran Santiago fueron más altas, pero desde entonces hasta 2002 han sido superadas por las de algunas ciudades medias como Iquique, La Serena, Puerto Montt y otras.

Tabla 2. Tasas de crecimiento de la población, 1930-2002.

\begin{tabular}{|c|c|c|c|c|}
\hline \multirow{2}{*}{ Periodo } & \multicolumn{4}{|c|}{ Tasa de crecimiento constante (r\%) } \\
\cline { 2 - 5 } & Chile & $\begin{array}{c}\text { La } \\
\text { Serena }\end{array}$ & $\begin{array}{c}\text { Gran } \\
\text { Santiago }\end{array}$ & $\begin{array}{c}\text { Puerto } \\
\text { Montt }\end{array}$ \\
\hline $1930-40$ & 1,6 & 0,9 & 3,6 & 2,6 \\
$1940-52$ & 1,4 & 2,3 & 3,8 & 0,9 \\
$1952-60$ & 1,8 & 2,6 & 3,6 & 2,4 \\
$1960-70$ & 1,9 & 2,3 & 3,2 & 2,5 \\
1970,82 & 2,0 & 2,3 & 3,3 & 1,4 \\
$1982-92$ & 1,7 & 2,4 & 2,0 & 2,3 \\
$1992-02$ & 1,2 & 2,8 & 1,3 & 3,1 \\
\hline
\end{tabular}

Fuente: Elaboración propia a partir de los censos de población INE. Tasa de crecimiento constante $(r \%)=\left(\right.$ raiz $\left.t\left\langle P_{t+n} / P_{t}\right)\right)-1 P_{t+n}$ : población media de un período iniciado en el año t y de $n$ años de duración; $\mathrm{N}$ : años transcurridos entre $\mathrm{t}$ y $\mathrm{t}+n$.

La "explosión" demográfica de las ciudades se debe, más que al crecimiento natural de la población, a los movimientos migratorios campo-ciudad. Los nuevos habitantes se han instalado en los márgenes del tejido urbano, en poblados planificados o en asentamientos espontáneos, y también en determinados sectores (los más baratos y deteriorados) del tejido urbano tradicional.

Los elevados valores de los movimientos intraurbanos, que superan a la dirección tradicional, son más recientes. En la Región Metropolitana de Santiago estos flujos residenciales, cuya dirección neta es desde el centro a la periferia, han involucrado a más de un millón de personas entre 1997 y 2002. La caracterización de estas migraciones se puede encontrar en Ortiz y Escolano (2004), pero para los objetivos de este trabajo conviene destacar lo siguiente: 
- En relación con la distancia, cabe distinguir los desplazamientos más largos, que se originan en las comunas del centro y acaban en las periféricas (SantiagoMelipilla; Santiago-San Bernardo; Las Condes-Colina), de los movimientos más cortos que se producen entre comunas vecinas o próximas (La Florida-Puente Alto; Las Condes-Providencia). Los segundos son más voluminosos que los primeros.

- Se observa una gran concentración en los destinos: Puente Alto, Maipú, Quilicura y San Bernardo han recibido desde 1997 hasta 2002 entre 10.000 y 60.000 inmigrantes metropolitanos cada una.

- El origen de los migrantes registra cierta dispersión, aunque algunas comunas presentan altos valores absolutos y relativos. La Florida, Conchalí, Santiago, Recoleta, Cerro Navia y La Granja, pierden más de 10.000 personas cada una en el mismo período.

- La trama compuesta por todos los movimientos intercomunales es densa y complicada. Algunas comunas, generalmente vecinas, forman "pares reflejos", y cada una emite y recibe emigrantes de la otra (Las Condes-Vitacura; ÑñoaPeñalolén), otras muestran preferencias en los destinos (Buin por Paine; Cerrillos por Maipú), mientras que unas terceras los diversifican (La Florida; Maipú; Santiago).

Como ya se ha apuntado, los datos censales no permiten realizar análisis a escala intraurbana en La Serena y Puerto Montt, ya que su espacio urbano se desarrolla, prácticamente, dentro del término municipal. No obstante, informaciones obtenidas en trabajos de campo y las derivadas del estudio de la evolución de la población por zonas y distritos, abonan la existencia de pautas espaciales de las migraciones intraurbanas similares a las expuestas para la región de Santiago. En La Serena, los distritos centrales (Intendencia, F. de Aguirre; Mercado) registran tasas de crecimiento demográfico negativas desde 1982 (entre $-0,7 \%$ y $-2,1 \%$ ), frente a las positivas de las áreas periféricas del norte (Las Compañías: $5,3 \%$ ) y del sur y sureste (Las Vegas: 3,5\%, La Pampa: 2,1\%). (Escolano y Ortiz, 2003). En Puerto Montt no hemos medido estas variaciones, pero las tendencias son idénticas.

La salida de la población hacia la periferia próxima y lejana de las ciudades se relaciona con el crecimiento de las viviendas, para primera y segunda residencia. La tasa de crecimiento de las viviendas es más alta que la de la población, por lo que desciende el número de personas por vivienda y se rebajan también otras ratios como la de personas/parcela, personas/m2 construido y personas/habitación. Si a este consumo de suelo se añade el ocupado por las infraestructuras, por los usos productivos y los espacios abandonados, se tienen los ingredientes que definen el modelo de crecimiento urbano disperso. 
Tabla 3. Evolución de la población y de las viviendas en las áreas urbanas, 1982-2002.

\begin{tabular}{|l|c|c|c|}
\hline \multirow{2}{*}{ Variables } & \multicolumn{3}{|c|}{ Área urbana } \\
\cline { 2 - 4 } & La Serena & Santiago & Puerto Montt \\
\hline 1982 & 83.238 & 3.882 .100 & 84.410 \\
1992 & 109.293 & 4.729 .118 & 110.139 \\
2002 & 160.148 & 5.408 .150 & 175.938 \\
Crecim. (\%) 1982-2002 & 92,4 & 39,3 & 108,4 \\
\hline Viviendas & & & \\
1982 & 17.125 & 857.424 & 16.861 \\
1992 & 26.778 & 1.164 .278 & 24.832 \\
2002 & 43.223 & 1.262 .626 & 42.440 \\
Crecim. (\%) 1982-2002 & 152,4 & 47,2 & 151,7 \\
\hline Personas/vivienda & & & 5,0 \\
1982 & 4,9 & 4,5 & 4,4 \\
1992 & 3,7 & 4,1 & 4,1 \\
2002 & $2002: \approx 1.800(2)$ & Ciudad compacta: $\approx 104.569(2)$ & $1985: 1.253(3)$ \\
Superficie urbana & Crec. $1991-00: \approx 400(2)$ & Crec. 1998-2003:16.828(1) & $2002: \approx 2.473(2)$ \\
(hectáreas) & & 4,3 & 5 \\
\hline
\end{tabular}

Fuente: Población y viviendas: Censos del INE.

Superficie urbana. (1): Mapa de usos del suelo del CONAF; (2): Cálculos propios a partir de fotogramas aéreos; (3) Varela, C. (2003).

\subsection{La reorganización espacial de las densidades: expansión y dispersión}

El crecimiento de la población y del espacio construido ha ido acompañado de cambios radicales en la estructura de la distribución espacial de la población. El modelo tradicional, caracterizado por la emergencia de las densidades más altas en el centro histórico que descienden, de forma más o menos suave, hacia la periferia, ha evolucionado hasta otro, cuya nota distintiva se define por la presencia de varias áreas de densidades máximas, excéntricas y discontinuas.

Las magnitudes fundamentales de los nuevos modelos se recogen en la tabla 4 , las propiedades más importantes de su configuración espacial se describen a continuación (figuras 1 y 2).

1. La densidad media de la población evidencia una tendencia sostenida al descenso, al menos desde la década de los 60 del pasado siglo. En Puerto Montt hemos podido reconstruir una serie de valores que permite medir la intensidad y ritmo de la caída: desde los $120 \mathrm{hab}$./ha en 1960 se ha pasado a los 63 hab./ha en 2002 (Escolano y Ortiz, 2004; en prensa). Para La Serena y Santiago no disponemos de datos para establecer comparaciones temporales, pero las trayectorias son parecidas y el retroceso puede ser, incluso, más acusado que el de Puerto Montt. 
Tabla 4. Principales características y parámetros de los modelos de densidad.

\begin{tabular}{|l|c|c|c|}
\hline Caracteristicas & La Seran & $\begin{array}{c}\text { Santiago } \\
\text { (ciudad compacta) }\end{array}$ & Puerto Montt \\
\hline Parametros del filtro & & & \\
-Puntos de la muestra & 207 & 449 & 1.659 \\
-Radio del kernel (m) & 500 & 2.000 & 300 \\
-Resolución (m) & 15 & 100 & 15 \\
\hline Densidad (hab./ha) & 148 & 237 & 297 \\
-Máxima (2002) & 82 & 45 & 63 \\
-Media (2002) & 1,65 & 1,06 & 0,80 \\
\hline Compacidad (2002) & 2.635 & 10.897 & 2.250 \\
\hline -Rho & & & \\
-Distancia agregada al centro (m) & & & \\
\hline
\end{tabular}

2. La organización espacial de la densidad configura una planta fragmentada y compleja, con varios núcleos de densidad máxima, más cercanos a los márgenes del tejido urbano continuo que al centro: el modelo de densidades de la ciudad compacta tradicional se ha cuarteado.

En La Serena, las densidades más elevadas se disponen en una banda discontinua con alguna extensión lateral, que sigue las vías de comunicación principales: arranca, por el norte en el centro de Las Compañías (135 hab./ha) para proseguir, tras el vacío del cajón del río Elqui, por el centro histórico $(92 \mathrm{hab} / \mathrm{ha})$, desde aquí se bifurca hacia el oriente siguiendo la ruta 41 , donde se alcanza una de las cumbres de las densidades de La Serena (147 hab./ha); hacia el sur la cresta de máximos continua, más atenuada, por Balmaceda y la ruta 43, hasta la Avda. de las Cuatro Esquinas, en el entorno de cuya intersección se encuentra otro máximo de densidades (148 hab./ha). Alrededor de esta espina dorsal las densidades descienden hacia la periferia, donde se localizan los valores más bajos.

La disposición de la topografía de las densidades del Gran Santiago es la más compleja. Las densidades máximas conforman varios arcos, alineaciones y relieves aislados que bordean el centro histórico, recogiendo la influencia de las vías de comunicación y de los usos y valor del suelo. Al sur se despliega un "macizo" que arranca de en la comuna del Bosque (215 hab./ha) y sigue, a través de San Ramón, La Granja y La Florida, hasta Puente Alto, donde se encuentra la cima de las densidades del Gran Santiago (237 hab./ha). Una dorsal, con dirección NE-SE, ocupa el occidente urbano, desde Huechuraba, por Renca, Cerro Navia y Pudahuel hasta Maipú (entre 140 y 180 hab./ha); un "relieve residual" de esta alineación es la prominencia de Quilicura (175 hab./ha). Finalmente, una cuña, no muy compacta, de densidades relativamente altas se extiende por el nororiente de la ciudad: por Providencia, Las Condes, y La Reina para culminar en Peñalolén (175 hab./ha). Estas tres áreas se 
hallan separadas por otros tantos "valles" y "desfiladeros" que confluyen en el centro histórico, cuyas densidades son más modestas (55 hab./ha). Las densidades por debajo de este valor se encuentran en los márgenes del espacio construido y en los fragmentos periféricos.

En Puerto Montt las densidades se ordenan en torno a varias modas espaciales, mientras que el distrito central y la periferia presentan valores más bajos. Los máximos se disponen en una semicorona discontinua, con algún espolón lateral, en torno al centro histórico o en los márgenes de alguna vía de comunicación importante: arrancan, en el poniente en los poblados del distrito de Mirasol donde se halla la cima de las densidades urbanas (297 hab./ha), para proseguir, hacia el norte, por fragmentos del distrito de Angelmó; tras el vacío del parque industrial, los valores ascienden en el distrito de Chinchín ( $225 \mathrm{hab} / \mathrm{ha}$ ), desde aquí, descienden de nuevo hacia el oriente, para remontar otra vez en La Paloma, donde se inflexionan hacia el sur (175 hab./ha), para terminar, después de la marcada ruptura del estero Lobos, en Pelluco (180 hab./ha). Los "valles" y "desfiladeros" que fragmentan esta alineación de densidades máximas coinciden con las áreas de fuertes pendientes de los escarpes de las terrazas y otros desniveles topográficos (quebradas, ríos), y con los usos industriales del suelo u ocupados por los principales ejes de transporte.

La configuración espacial de las densidades se halla condicionada tanto por las vías de comunicación y el valor y usos del suelo como por la presencia de elementos físicos (costas, ríos, áreas de fuertes pendientes), como se aprecia, claramente en Puerto Montt y La Serena. Todos los modelos se asemejan a una onda o a un volcán, cuya planta puede ser semicircular, como en Puerto Montt, o netamente circular como en Santiago, o adquiere la forma de elipse muy alargada como en La Serena. Sin embargo, por encima de las particularidades de cada ciudad, la estructura de los modelos es muy similar, especialmente los de Santiago y Puerto Montt (figura 3). La Serena, además de valores más bajos de densidad presenta ligerísimas diferencias de estructura con los anteriores.

3. El aumento de la extensión del espacio urbano ha ido emparejado con la merma de su compacidad: los límites urbanos no sólo se han tornado más difusos, sino también más sinuosos, lo que se agrava con la aparición de islotes de tejido urbano en la periferia, que ensartados por las vías de comunicación forman un denso archipiélago en torno a la ciudad tradicional. El índice rbo, que mide la compacidad de la densidad respecto de una figura ideal (un cilindro de densidad constante), supera la unidad en Santiago y arroja un valor de dispersión considerable en La Serena $(1,65)$, Puerto Montt mantienen todavía un grado de compacidad aceptable. $R b o$ es un indicador sintético de eficiencia de la ciudad, estimada por la distancia agregada que la población ha de recorrer para desplazarse al centro funcional; esa longitud, expresada en unidades métricas, es de $2,6 \mathrm{~km}$ en La Serena, $10,9 \mathrm{~km}$ en Santiago y $2,2 \mathrm{~km}$ en Puerto Montt. 


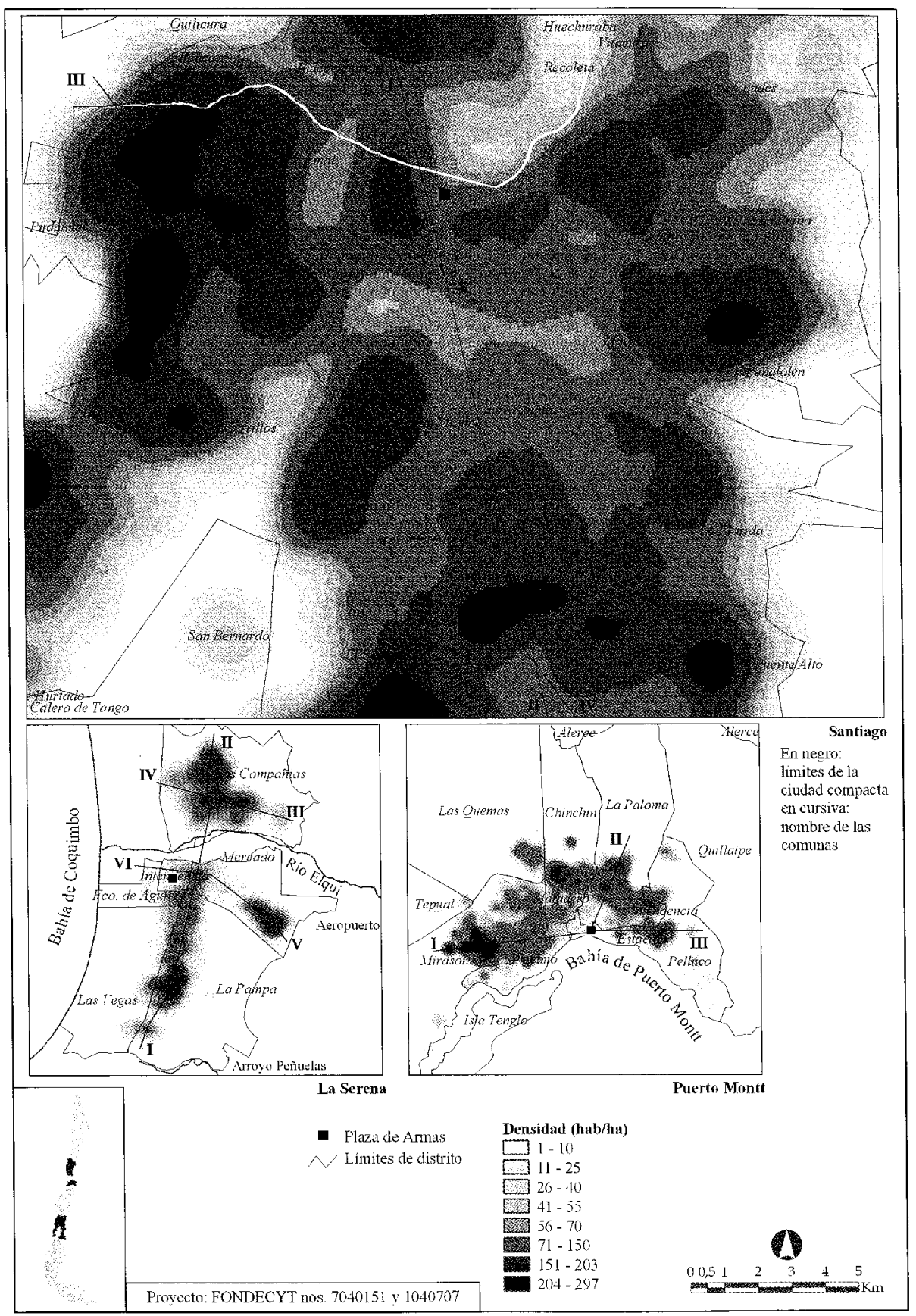

Figura 1. Densidad de población en 2002 (habitantes/ha) (Método: kernel). 


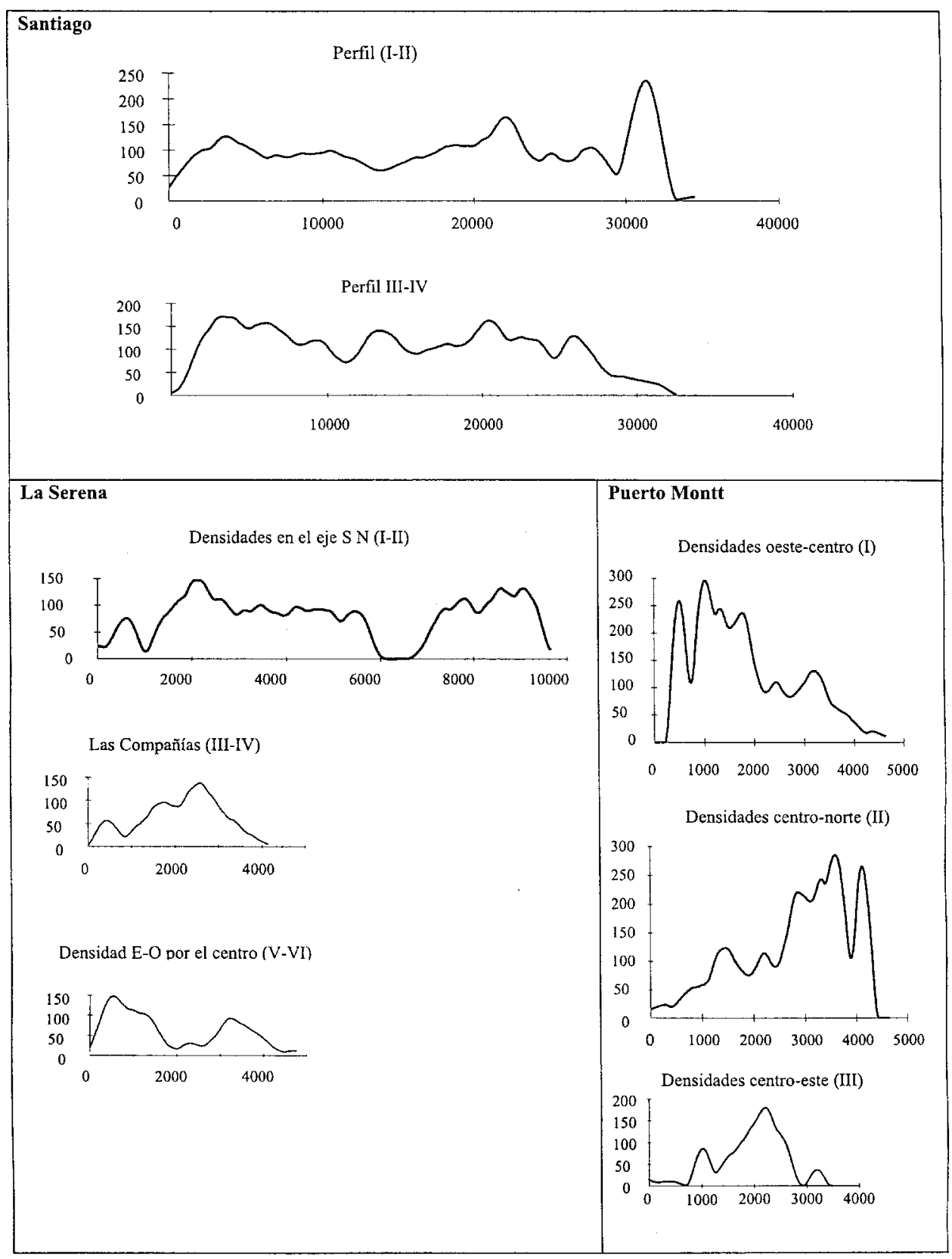

Figura 2. Perfiles de la densidad de población en 2002. (Habitantes/ha). (La localización de los perfiles se encuentra en la figura 1. Eje y: densidad; eje $\mathrm{x}$ : distancias en $\mathrm{m}$ Los gráficos de Santiago no guardan proporción en las distancias con los de las otras ciudades). 


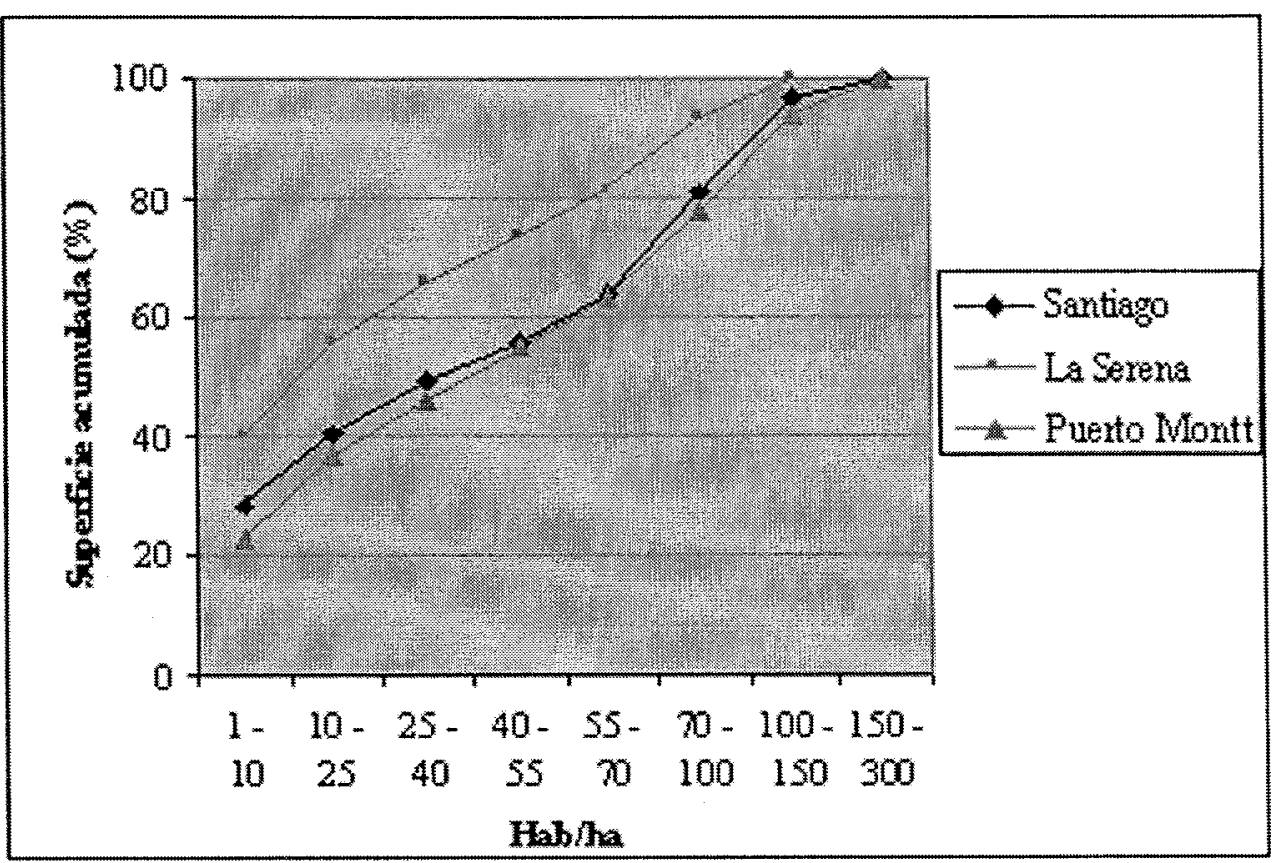

Figura 3. Estructura de la distribución de las densidades según la superficie ocupada. 2002.

En resumen: el descenso de la densidad y la consolidación de un nuevo modelo de densidades se constata en las tres ciudades objeto de estudio. La intensidad de su caída es más fuerte en La Serena que en la ciudad compacta de Santiago y en Puerto Montt.

\section{Consideraciones finales: crecimiento de la dispersión y de la ineficiencia de las ciudades}

El nuevo modo de producción de espacio urbano, así como los modelos socioespaciales, morfológicos y funcionales asociados, introducen cierto grado de ruptura con el espacio urbano preexistente, al menos en el paisaje y en la estructura espacial de la densidad de población.

La densidad es el indicador por excelencia de la intensidad de ocupación del suelo y forma parte de todos los sistemas de indicadores de sostenibilidad urbana. Aunque 
no se ha demostrado de forma directa e incontestable la relación entre la densidad y la sostenibilidad, si que se ha probado la asociación entre valores medios y altos de densidad y menor numero de desplazamientos urbanos en vehículo privado, y en consecuencia menor consumo de combustible, descenso de la contaminación atmosférica, mejores aprovechamientos energéticos en la edificación... Los polos compacto-disperso no son, seguramente, ni deseables ni prácticos; en cambio, una combinación adecuada de concentración y dispersión, fomentando densidades aitas en torno a los ejes de comunicaciones y rebajândolas en otros lugares, es más útil y alcanzable.

En las ciudades estudiadas es posible que sea necesario atajar la ineficiencia e detener la dispersión en La Serena y en Santiago, mientras que en Puerto Montt, aunque se confirma la tendencia a la disgregación, todavía es posible orientar de forma apropiada el crecimiento urbano conservando los valores de todo tipo que aún posee como núcleo compacto.

\section{Bibliografía}

Arriagada, C. (2000) Pobreza e América Latina: Nuevos escenarios $y$ desafios de politicas para el bábitat urbano. Vol. 27, LC/L.1429-P, Cuadernos de la CEPAL. Santiago de Chile.

Arriagada, C., y Rodríguez J. (2003) Segregación residencial en áreas metropolitanas de América Latina: magnitud, caracteristicas, evolución e implicaciones de politica. Vol. 47, LC/L1997-P, Población y Desarrollo, CEPAL, Santiago de Chile.

Balbo, M., Jordán R., y Simini, D. (eds.) (2003) La ciudad inclusiva. Vol. 88, LC/G.2210-P, Cuadernos de la CEPAL. Santiago de Chile.

Bertaud, A., y Malpezzi, S. (1999) The Spatial distribution of Population in 35 World Cities: The Role of Markerts, Planning and Topography. (vía URL).

Clichevsky, N. (2000) Informalidad y segregación urbana en América Latina. Una aproximación. Vol. 28, LC/L.1430-P, Medio Ambiente y Desarrollo, CEPAL, Santiago de Chile
Escolano, S. (2002) Densidad de población y sustentabilidad en la ciudad de Zaragoza. En Aportaciones geográficas en memoria del Profesor L. Miguel Yetano Ruiz, edited by J.L. Peña and L.A Longares, 173-82, Universidad de Zaragoza, Zaragoza.

Escolano, S., y J. Ortiz (2004) La complejidad de los procesos de reestructuración socioespacial en las ciudades intermedias: persistencia y cambio en la ciudad de Puerto Montt (Chile). Anales de Geografía de la Universidad Complutense, $n^{\circ} 24,79-106$

Escolano, S. y Ortiz, J. (2003) Procesos de reestructuración urbana en ciudades intermedias del sistema urbano chileno: el caso de La Serena en la Región IV de Coquimbo. Actas del 51 Congreso Internacional de Americanistas, Santiago de Chile.

Kaztman, R. (2003) La dimensión espacial en las politicas de superación de la pobreza urbana. Vol. 59, LC/L.1790-P, Media Ambiente y Desarrollo, CEPAL, Santiago de Chile. 
Mattos, C. de (2002) Transformaciones de las ciudades latinoamericanas impactos de la globalización? Eure XXVIII, $n^{\circ} .85,5-$ 10.

Mertins, G. (2000) Ciudades medianas de América Latina: criterios, indicadores y el intento de un modelo de diferenciación socio-espacial y funcional. Espacio $y$ Desarrollo, 13-23.

Naciones Unidas (2003) World Urbanization Prospects. The 2003 Review, Data, Tables and Highlights. Nueva Cork.

Ortiz, J., y S. Escolano (2004) Patrones recientes de movilidad espacial de la población del Gran Santiago. 1997-2002. Actas del $X X V$ Congreso Nacional $y X$ Internacional de Geografia, Valdivia (Chile).

Pinto da Cunha, J.M. (2002) Urbanización, redistribución espacial de la población y transformaciones soioeconómicas en America Latina. Vol. 30, LC/L. 1782-P, Población y Desarrollo, CEPAL, Santiago de Chile.
Prevot, M.F. (1999) Amérique Latine: la ville fragmentée. Esprit, n 258.

Sabatini, F., G. Cáceres, y J. Cerdá (2001) Segregación residencial en las principales ciudades chilenas. Tendencias en las tres últimas décadas y posibles cursos de acción. Eure, n²7 (82), 21-42.

Thurstain-Goodwin, M. (2003) Data surfaces for a new policy geography. En: Longley, P. A. y Batty, M. (eds.) Advanced spatial analisis. The CASA book of GIS, 145-70, Esri Press, Redlands.

Varela, C. (2003) Procesos de cambio socioespaciales y funcionales en una ciudad intermedia chilena: Puert Montt, X Región de Los Lagos, Chile. Actas del 51 Congreso Internacional de Americanistas, Santiago de Chile.

Véliz, G. (1995) Conurbación La SerenaCoquimbo. Editorial Rosales Hnos. Ltda., La Serena. 